hep-th/0006181

PAR-LPTHE 00-26

ESI-907

June 2000

\title{
SOLITONIC SECTORS, $\alpha$-INDUCTION AND SYMMETRY BREAKING BOUNDARIES
}

\author{
Jürgen Fuchs ${ }^{1}$ and Christoph Schweigert ${ }^{2}$ \\ 1 Institutionen för fysik \\ Universitetsgatan 1 \\ S-651 88 Karlstad \\ 2 LPTHE, Université Paris VI \\ 4 place Jussieu \\ F-75 252 Paris Cedex 05
}

\begin{abstract}
We develop a systematic approach to boundary conditions that break bulk symmetries in a general way such that left and right movers are not necessarily connected by an automorphism. In the context of string compactifications, such boundary conditions typically include non-BPS branes.

Our formalism is based on two dual fusion rings, one for the bulk and one for the boundary fields. Only in the Cardy case these two structures coincide. In general they are related by a version of $\alpha$-induction. Symmetry breaking boundary conditions correspond to solitonic sectors. In examples, we compute the annulus amplitudes and boundary states.
\end{abstract}




\section{Introduction}

Conformally invariant boundary conditions of two-dimensional conformal field theories arise in the study of defects in systems of condensed matter physics, of percolation probabilities and of (open) string perturbation theory in the background of D-branes. They are presently under active investigation. Boundary conditions that preserve all bulk symmetries for theories with charge conjugation modular invariant have been treated by Cardy [1]. The two basic results are the following: Boundary conditions are labelled by the primary fields of the theory, and the annulus multiplicities are given by the fusion rules. Together with the information that all bulk symmetries are preserved, these two results allow in particular to recover the so-called boundary states, which encode all one-point amplitudes on the disk.

More recently, these results have been generalized in several directions [2,3, [4. In particular, those boundary conditions have been classified which preserve an abelian orbifold subalgebra of the algebra $\mathfrak{A}$ of bulk symmetries, i.e. for which the preserved symmetries can be characterized as the subalgebra $\overline{\mathfrak{A}}=\mathfrak{A}^{G}$ of symmetries that are fixed by some abelian group $G$ of automorphisms of $\mathfrak{A}$. Boundary states for such boundary conditions have been given explicitly, and the integrality of the annulus coefficients was proven. It was also shown that correlation functions in the presence of such boundary conditions can be written as linear combinations of twisted conformal blocks. As a special case, the boundary states can be expressed in terms of twisted Ishibashi states $|\lambda, \omega\rangle\rangle$, which are characterized by the identity

$$
\left.\left(Y_{n} \otimes \mathbf{1}+(-1)^{\Delta_{Y}-1} \mathbf{1} \otimes \omega\left(Y_{-n}\right)\right)|\lambda, \omega\rangle\right\rangle=0
$$

for every primary field $Y$ (of conformal weight $\Delta_{Y}$ ) in the chiral symmetry algebra $\mathfrak{A}$. Here $\omega \in G$ is an automorphism of the bulk symmetries that leaves the Virasoro algebra invariant, $\omega\left(L_{n}\right)=L_{n}$. In a sense, the relations (11) express the fact that at the boundary left movers and right movers are connected by the automorphism $\omega$. The automorphism $\omega$ has been called the automorphism type, or gluing automorphism, of the boundary condition. We will say that such boundary conditions possess a definite automorphism type, in this case $\omega$.

In the present letter, we study more general patterns of symmetry breaking by boundaries, in which left movers and right movers are not necessarily related any more by automorphisms. In more precise terms, this means that the boundary conditions preserve a subalgebra $\overline{\mathfrak{A}}$ of the algebra $\mathfrak{A}$ of bulk symmetries that cannot any longer be characterized as a fixed algebra under some group of automorphisms. We refer to such boundary conditions as boundary conditions without automorphism type, or without gluing automorphism. Examples of such boundary conditions appear already for the $\mathbb{Z}_{2}$-orbifold of a compactified free boson. Other examples are provided by various conformal embeddings; boundary conditions associated to conformal embeddings have been studied in [5, 6], in particular in their relation with certain graph algebras [7, 8, 9, 10].

Boundary conditions without automorphism type are of direct relevance in string theory: they correspond to non-BPS branes. Indeed, every chiral algebra automorphism $\omega$ maps the vertex operator of a space-time supercharge to the vertex operator of another supercharge.

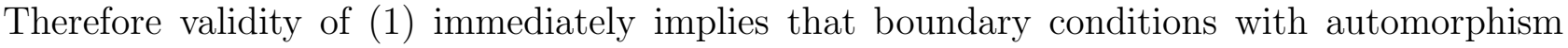
type preserve half of the space-time supersymmetries, and hence are BPS.

The purpose of the present note is to generalize Cardy's results and those of [2, [3, [] once more. We consider conformally invariant boundary conditions of a rational conformal field 
theory with chiral algebra $\mathfrak{A}$ that preserve a subalgebra $\overline{\mathfrak{A}}$ of $\mathfrak{A}$ that is still rational, but otherwise arbitrary. We give a natural labelling of the boundary conditions and compute the annulus coefficients. By a modular transformation, this allows to determine the boundary states.

For certain conformal field theories, one can construct (nets of) factors; their irreducible local sectors (inner unitary equivalence class of representations) are in one-to-one correspondence to the primary fields of the conformal field theory. Our main tool is an adaptation of a certain form of induction for sectors, the so-called $\alpha$-induction, which was developped [11, 12, 13, 14, 15] in the framework of subfactor theory. Applying $\alpha$-induction to a sector of the subfactor, it produces a sector of the ambient factor. Among the sectors obtained this way there are ordinary, local, sectors as well as solitonic sectors.

For the purposes of this letter, we do not have to know the relevant nets of subfactors and their sectors in any detail. Rather, we simply postulate that the process of $\alpha$-induction works at the level of the representation category of the conformal field theory under investigation. Thus we regard the irreducible sectors as the primary fields, or rather as the associated basis elements of the fusion ring; general sectors correspond to arbitrary elements of the fusion ring, and the composition of sectors is simply the fusion product. In fact, all we need to know is the action of $\alpha$-induction on primary fields. It will provide us with solitonic sectors which precisely label symmetry breaking boundary conditions. Moreover, the fusion of these sectors will provide us with the annulus multiplicities. As in the Cardy case, these data, together with the preserved symmetries $\overline{\mathfrak{A}}$, allow to construct the boundary states.

In section 2 we discuss the labelling problem for bulk and boundary fields. Motivated by constructions from topological field theory, we are led to the concepts of bulk and boundary categories. Our prescription for the boundary category is presented in section 3. It is based on imposing a version of $\alpha$-induction at the level of the fusion rules. In section 4 two illustrative examples are analyzed.

\section{Symmetry breaking boundary conditions}

Before we explain the case of boundary conditions without automorphism type, we briefly rephrase some of the results of [3, [4] on boundary conditions that do possess an automorphism type. As was shown there, boundary conditions leaving $\overline{\mathfrak{A}}=\mathfrak{A}^{G}$ invariant correspond to orbits $[\bar{\mu}, \psi]$ of primary fields $\bar{\mu}$ of the $\overline{\mathfrak{A}}$-theory with respect to a group $\mathcal{G} \cong G^{*}$ of simple current fields (the degeneracy label $\psi$ is a character of a suitable subgroup of $\mathcal{G}$ ). The monodromy charge [16] of $\bar{\mu}$ with respect to $\mathcal{G}$ is not restricted. These labels $[\bar{\mu}, \psi]$, in turn, can be seen to correspond to representations of $\mathfrak{A}$; these are twisted representations when the monodromy charge of $\bar{\mu}$ is not zero. T Twisted representations of vertex operator algebras have been investigated e.g. in 17, 18, 19, 20]. The notion of fusion of such representations has also been studied [21, but little is known about the resulting fusion ring. However, one can re-write the annulus amplitudes derived in [3] as sums of characters of the twisted representations. It is therefore reasonable to expect that the annulus coefficients determined in [3] precisely coincide with the fusion rules of the twisted representations.

A second ingredient we will need is the description of correlation functions in the presence

\footnotetext{
${ }^{1}$ When the action of the twists is only projective, additional subtleties arise.
} 
of boundaries through three-dimensional topological field theory that was developped in [22,23] for symmetry preserving boundary conditions. In that context, a three-dimensional manifold $M_{X}$, the connecting manifold, was constructed to compute correlators on a two-dimensional world sheet $X . M_{X}$ has a boundary, and this boundary is isomorphic to the so-called double $\hat{X}$ of $X$. The connecting manifold is universal in the sense that it is the same for all rational conformal field theories. One also needs to prescribe a Wilson graph in $M_{X}$. Bulk points on the world sheet $X$ possess two pre-images on its double $\hat{X}=\partial M_{X}$, and these two points are connected by a natural interval in $M_{X}$. A Wilson line carrying the bulk label is placed in that interval.

As for the boundary data, a circular Wilson line must be placed parallel to each boundary component. Insertions of boundary fields are linked with short Wilson lines to the corresponding circular Wilson line. This is summarized in the following picture for the case when $X$ is the disk (then $\hat{X}$ is the sphere and $M_{X}$ is a solid three-ball):

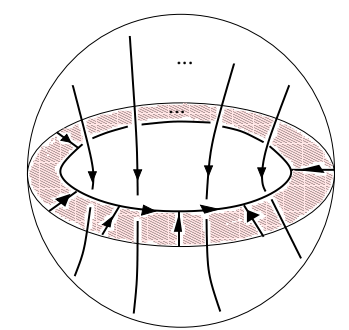

We will assume that the Wilson graph is universal as well, i.e. that the same graph is still to be used for symmetry breaking boundary conditions. The boundary graph must be labelled, too. In the Cardy case, we use the same type of labels as for the bulk components, i.e. primary fields of $\mathfrak{A}$. This nicely fits together with Cardy's result that symmetry preserving boundary conditions are in one-to-one correspondence with primary fields. Moreover, in this description trivalent vertices, couplings, appear naturally in the boundary graph. They involve two boundary conditions and the chiral label of a boundary insertion.

We remark that typically the Wilson graph is not connected. What is crucial in the present context is that there is never a Wilson line that connects a bulk insertion with a boundary insertion or with a segment of the Wilson graph that encodes a boundary condition. We are thereby led to the following general picture. Boundary conditions as well as field insertions on the boundary should be characterized by basis elements of the same 'fusion ring', and the structure constants of this fusion ring should coincide with the annulus coefficients. In more technical terms, the labels should correspond to the (isomorphism classes of) simple objects of a suitable tensor category. We call this structure the boundary category. Bulk fields, on the other hand, will have to be described by a different structure, namely as the simple objects of a bulk category; their treatment is beyond the scope of the present note. Notice that the interpretation in terms of boundary insertions requires that associated to each boundary label there comes a natural state space, which is a module over (at least) the Virasoro algebra.

In Cardy's case - torus partition function of charge conjugation type, and only symmetry preserving boundary conditions - both the boundary and the bulk category are just the one associated to the fusion ring of the full bulk symmetry $\mathfrak{A}$, and the state space associated to a label $\lambda$ is nothing but the corresponding irreducible $\mathfrak{A}$-module $\mathcal{H}_{\lambda}$. For a general boundary insertion, the state space must be an appropriate generalization of a twisted representation, or in other words, a solitonic [24, 25, 26] sector. In Cardy's case, the tensor category has 
actually a lot more structure. In particular it is modular [27, i.e., roughly speaking, there is a braiding [28, 29], leading to the unitarity of the modular matrix $S$, as well as a twist of Wilson lines, which corresponds to the modular matrix $T$.

This is too much structure than may be expected to be present for a boundary category in the general case. As examples of twisted representations show, the twist of a Wilson line will, in general, no longer be a well-defined operation on the boundary. Correspondingly, the boundary sectors will not have a unique conformal weight up to integers. (The bulk category, on the other hand, will still possess a $T$-matrix.) Similarly, neither the bulk nor the boundary category will possess a braiding any more, in general. However, it is still possible to braid an object of the bulk category with an object of the boundary category. This braiding allows us to establish a generalization of the diagonalizing matrix $\tilde{S}$ that was introduced in [3]. We expect that this matrix $\tilde{S}$ is square; nevertheless its two indices take values in two different sets: the rows are labelled by the bulk fields, whereas the columns are labelled by the boundary conditions. Up to normalization, $\tilde{S}$ provides the coefficients for the expansion of the boundary states with respect to the boundary conformal blocks (generalized Ishibashi states). We conjecture that the matrix $\tilde{S}$ obtained this way is invertible and hence in particular indeed a square matrix (and can be chosen unitary). This implies that the bulk and the boundary category have the same number of simple objects; as a consequence, the number of boundary conditions can be read off from the bulk modular invariant. The equality between the number of bulk fields and the number of boundary conditions has been derived in [3] for boundary conditions with definite automorphism type; we will see in the examples below that it holds for more general symmetry breakings as well.

\section{Solitonic sectors from $\alpha$-induction}

According to the reasoning above, we would like to view the boundary labels $\mu$ as simple objects of a suitable category, and accordingly the associated intertwiners as morphisms of that category. It will, however, be important that we can regard the sectors also as (isomorphism classes of) representations - including both ordinary and solitonic representations - of the chiral algebra or vertex operator algebra $\mathfrak{A}$. These representation spaces provide the spaces of open string states whose partition function is the annulus amplitude.

How can we then obtain the boundary category? We wish to find 'solitonic' $\mathfrak{A}$-representations. Fortunately, there is one situation in which this can be done totally explicitly. Namely, chiral WZW theories can also be analyzed in the framework [30] of nets of operator algebras. In that context, one can employ the notion of $\alpha$-induction to arrive at solitonic sectors of the chiral theories [11, [13].

The concrete construction of operator algebras for more general chiral conformal field theories is a difficult problem; see [31, 32], as well as [33, 34] for the case of coset and orbifold theories. For the purposes of the present note, we need not address these questions which are definitely quite important. Rather, we will only abstract from $\alpha$-induction and its adjoint operation, $\sigma$-restriction, a few properties at the level of fusion rings. We present these properties in the form of a recipe. (But we expect that they are indeed realized in any decent conformal field theory, and actually that the existence of such an induction procedure can be entirely established in the context of the relevant tensor categories.)

We start by prescribing those bulk symmetries that are preserved by the boundary condi- 
tions. These symmetries must form a consistent rational subalgebra $\overline{\mathfrak{A}}$ of the chiral algebra $\mathfrak{A}$. We assume that the collection of all (isomorphism classes of) ordinary - i.e. non-solitonic - irreducible modules $\overline{\mathcal{H}}_{\bar{\lambda}}$ over $\overline{\mathfrak{A}}$ gives rise to a modular fusion ring with basis $\left\{\bar{\Phi}_{\bar{\lambda}}\right\}$. From the embedding of $\overline{\mathfrak{A}}$ into $\mathfrak{A}$ we determine how the vacuum module $\mathcal{H}_{\Omega}$ of $\mathfrak{A}$ decomposes into a direct sum of irreducible $\overline{\mathfrak{A}}$-modules:

$$
\mathcal{H}_{\Omega}=\bigoplus_{\bar{\mu}} b_{\bar{\mu}} \overline{\mathcal{H}}_{\bar{\mu}}
$$

(Thus $b_{\bar{\lambda}} \in \mathbb{Z}_{\geq 0}$ is the multiplicity with which the $\overline{\mathfrak{A}}$-module $\overline{\mathcal{H}}_{\bar{\lambda}}$ appears in the vacuum module of the $\mathfrak{A}$-theory.) This allows us to introduce the element

$$
\bar{\theta}:=\sum_{\bar{\mu}} b_{\bar{\mu}} \bar{\Phi}_{\bar{\mu}}
$$

of the fusion ring of $\overline{\mathfrak{A}}$; we refer to $\bar{\theta}$ as the extending sector of the $\overline{\mathfrak{A}}$-theory.

We now construct a new fusion ring as follows. First, it is generated by objects $\alpha_{\bar{\lambda}}$ for each basis element $\bar{\Phi}_{\bar{\lambda}}$ of the fusion ring of $\overline{\mathfrak{A}}$. The fusion product is defined by

$$
\alpha_{\bar{\lambda}} \star \alpha_{\bar{\mu}}:=\alpha_{\bar{\lambda} \star \bar{\mu}}
$$

and we also require that $\alpha_{\bar{\lambda} \oplus \bar{\mu}}=\alpha_{\bar{\lambda}}+\alpha_{\bar{\mu}}$ and $\alpha_{\bar{\lambda}^{+}}=\left(\alpha_{\bar{\lambda}}\right)^{+}$. This would not constitute anything new beyond what is encoded in the fusion ring of $\overline{\mathfrak{A}}$, were it not for another piece of information. Namely, the fusion ring element $\alpha_{\bar{\lambda}}$ is also supposed to represent a - possibly twisted or solitonic - representation of $\mathfrak{A}$, which for brevity we denote by the same symbol. An important point is that even for irreducible $\bar{\lambda}$ the $\mathfrak{A}$-representation $\alpha_{\bar{\lambda}}$ need not necessarily be irreducible, and that the $\alpha_{\bar{\mu}}$, respectively their irreducible subrepresentations, for different values of $\bar{\mu}$ are allowed to be isomorphic. (Thus in particular the $\alpha_{\bar{\lambda}}$ generically do not form a basis of the fusion ring.) In view of Schur's lemma, this information is conveniently encoded in the intertwiner spaces $\operatorname{Hom}_{\mathfrak{A}}\left(\alpha_{\bar{\lambda}}, \alpha_{\bar{\mu}}\right)$. For instance, $\alpha_{\bar{\lambda}}$ is irreducible if and only if $\operatorname{Hom}_{\mathfrak{A}}\left(\alpha_{\bar{\lambda}}, \alpha_{\bar{\lambda}}\right)$ is one-dimensional. Also, when $\alpha$ is a simple and $\beta$ any arbitrary object of the fusion category, then the dimension of $\operatorname{Hom}_{\mathfrak{A}}(\alpha, \beta)$ tells us how many times $\alpha$ appears in the decomposition of $\beta$. The Hom spaces are defined in terms of the intertwiner spaces in the fusion category of $\overline{\mathfrak{A}}$ as follows:

$$
\operatorname{Hom}_{\mathfrak{A}}\left(\alpha_{\bar{\lambda}}, \alpha_{\bar{\mu}}\right):=\operatorname{Hom}_{\overline{\mathfrak{A}}}\left(\bar{\Phi}_{\bar{\lambda}}, \bar{\theta} \star \bar{\Phi}_{\bar{\mu}}\right) .
$$

This system of Hom spaces obeys tight consistency constraints. For example, from $\operatorname{Hom}_{\mathfrak{A}}\left(\alpha_{\bar{\lambda}}\right.$, $\left.\alpha_{\bar{\lambda}}\right)$ we compute the number $n_{\bar{\lambda}}$ of irreducible subsectors of $\alpha_{\bar{\lambda}}$. If one would just prescribe an extending sector $\bar{\theta}$ at random, one might find contradictions of the type that more than $n_{\bar{\lambda}}$ irreducible sectors have non-trivial intertwiners with $\alpha_{\bar{\lambda}}$. The existence of a system of Hom spaces that is free of contradiction is therefore highly non-trivial and requires special properties of $\bar{\theta}$. A necessary condition is of course that all irreducible $\overline{\mathfrak{A}}$-subsectors of $\bar{\theta}$ are mutually local, but this condition is typically far from being sufficient. It would be rewarding to find a characterization of consistent extending sectors purely at the level of fusion rings. It will then be particularly interesting to compare the problem of classifying consistent extending sectors with the problem of classifying modular invariant partition functions of extension type.

It is also important that along with $\alpha$-induction there comes an "adjoint" operation, known as $\sigma$-restriction. Namely, every sector $\beta$ of $\mathfrak{A}$, whether solitonic or not, may be seen as a 
(typically reducible) sector of $\overline{\mathfrak{A}}$, which we denote as $\sigma(\beta)$. Induction and restriction are related by the reciprocity relation

$$
\operatorname{Hom}_{\mathfrak{A}}\left(\alpha_{\bar{\lambda}}, \beta\right) \cong \operatorname{Hom}_{\overline{\mathfrak{A}}}(\bar{\lambda}, \sigma(\beta))
$$

This implies that

$$
\sigma\left(\alpha_{\bar{\mu}}\right)=\bar{\theta} \star \bar{\mu}
$$

and allows us to decompose induced solitonic sectors into irreducible $\overline{\mathfrak{A}}$-sectors.

Let us pause and compare these ideas to the situation studied in [3]. In that case, $\bar{\theta}$ can be written as a sum over so-called simple current sectors $\bar{J}$ which form a finite abelian group $\mathcal{G}$ under fusion, and each such simple current appears with multiplicity one:

$$
\bar{\theta}=\sum_{\bar{J} \in \mathcal{G}} \bar{J} .
$$

Formula (6) then just summarizes how the fusion rules of a simple current extension are related to those of the original theory (in the category theoretical setting, this is discussed in [35, 36, 37]). However, it only allows for a direct determination of the extended fusion rules as long as no fixed points - that is, sectors $\bar{\lambda}$ with $\bar{J} \star \bar{\lambda}=\bar{\lambda}$ for some $\bar{J} \in \mathcal{G}$ - are involved. Indeed, in the simple current situation the induced sector $\alpha_{\bar{\lambda}}$ is reducible if and only if $\bar{\lambda}$ is a fixed point. The decomposition of $\alpha_{\bar{\lambda}}$ for a fixed point is precisely what is known as [16, 38 fixed point resolution in the theory of simple current extensions.

In the general case there is the following analogue of the problem caused by simple current fixed points. It can happen that the relations (6) do not provide enough information for decomposing all $\alpha_{\bar{\lambda}}$ into irreducible sectors. In that case, the category must be enlarged: sufficiently many additional irreducibles have to be introduced to provide subobjects. There exists a general procedure for doing so [39, 36]. But unfortunately fully explicit formulae, in particular for the modular $S$-matrix of the enlarged theory, are only known in the simple current case [38], where it leads in particular to the group character $\psi$ that appears in the description of boundary conditions with definite automorphism type, see above.

A more explicit understanding of these new irreducibles in the general case and in particular what their braiding properties with bulk fields are, might be called the generalized fixed point problem. To be precise, the task is to express the fusion products of the new irreducibles in terms of chiral data of the $\overline{\mathfrak{A}}$-theory, like e.g. the modular matrices for one-point conformal blocks on the torus. We consider this to be a central problem in the study of solitonic sectors, and hence of conformally invariant boundary conditions. I In the present letter, we restrict ourselves to examples where either this problem does not occur at all or where it can be resolved by using the knowledge about the simple current case.

Our prescription provides us explicitly with labels for the boundary conditions and the boundary insertions. The annulus multiplicities are just the tensor product multiplicities in the boundary category, and the open string states are organized in terms of the induced sectors. The induced sectors come in two classes: ordinary, non-solitonic sectors correspond to symmetry preserving boundary conditions, while the solitonic sectors are in correspondence

\footnotetext{
${ }^{2}$ A special version of the fixed point problem arises already when one aims to express the modular $S$-matrix of the $\mathfrak{A}$-theory through chiral data of the $\overline{\mathfrak{A}}$-theory. For exceptional extensions, no general solution to this problem is known.
} 
with symmetry breaking boundary conditions. In the case of boundary conditions with automorphism type, the latter are just the orbits with non-vanishing monodromy charge. In the subfactor framework, ordinary and solitonic sectors can be distinguished by their localization properties.

Before we support our findings by examples, we wish to add a speculative comment. In the operator-algebraic definition of $\alpha$-induction, a braiding among $\overline{\mathfrak{A}}$-sectors enters. In two dimensions there are two independent braidings - 'over'- and 'under'-braiding - which are each others' inverse. As a consequence, there are in fact two $\alpha$-inductions, called $\alpha^{ \pm}$. It has been shown in [13] that $\alpha_{\bar{\lambda}}^{ \pm}$is not solitonic if and only if $\alpha_{\bar{\lambda}}^{+}$and $\alpha_{\bar{\lambda}}^{-}$are isomorphic. This suggests that solitonic representations, and thus symmetry breaking boundary conditions, actually come in pairs. However, only one version of $\alpha$-induction may be used at a time; so there is a twofold choice on which set of (symmetry breaking) boundary conditions one must take. It will be interesting to see whether this can explain the observations in [40], where two distinct sets of symmetry breaking boundary conditions were found; any two boundary conditions of the same set are compatible, while two boundary conditions belonging to distinct sets are mutually incompatible.

\section{Examples}

Our general ideas are easily illustrated by examples; we present two of them. The first example is the $E_{6}$-type modular invariant of $A_{1}$ at level 10 , which has already been discussed extensively elsewhere [41, 12, 13, 6]. We will show how the structures developed above allow to rederive and systematize the results of [6] on the boundary conditions of this theory. The second example deals with the exceptional modular invariant of $G_{2}$ at level 3 and is, to the best of our knowledge, new.

The fusion ring of $A_{1}$ at level 10 has eleven simple sectors, which we label by $\bar{\mu}=0,1, \ldots, 10$. In this notation, the $E_{6}$-type modular invariant of $A_{1}$ reads

$$
Z=\left|\chi_{0}+\chi_{6}\right|^{2}+\left|\chi_{4}+\chi_{10}\right|^{2}+\left|\chi_{3}+\chi_{7}\right|^{2}
$$

It corresponds to the conformal embedding into $B_{2}$ at level 1 . The first block comes from the vacuum $o$, the second from the vector $v$ and the third block from the spinor $s$ of $B_{2}$. The relevant aspects of $\alpha$-induction for this example can be found in [13, Sec.2.2 of II]; here we summarize the most important features.

From the modular invariant (10) we read off the extending sector as $\bar{\theta}=\bar{\Phi}_{0}+\bar{\Phi}_{6}$. The dimensions of the Hom spaces are thus given by

$$
\begin{array}{r}
\operatorname{dim} \operatorname{Hom}_{\mathfrak{A}}\left(\alpha_{\bar{\mu}_{1}}, \alpha_{\bar{\mu}_{2}}\right)=\operatorname{dim} \operatorname{Hom}_{\overline{\mathfrak{A}}}\left(\bar{\Phi}_{\bar{\mu}_{1}}, \bar{\Phi}_{\bar{\mu}_{2}}\right)+\operatorname{dim} \operatorname{Hom}_{\overline{\mathfrak{A}}}\left(\bar{\Phi}_{\bar{\mu}_{1}}, \bar{\Phi}_{6} \star \bar{\Phi}_{\bar{\mu}_{2}}\right) \\
=\delta_{\bar{\mu}_{1}, \bar{\mu}_{2}}+\overline{\mathcal{N}}_{6, \bar{\mu}_{2}},
\end{array}
$$

where $\overline{\mathcal{N}}$ are the fusion rules of $A_{1}$ at level 10. Applying this to the case $\bar{\lambda}_{1}=\bar{\lambda}_{2}$, we find that the sectors $\alpha_{\bar{\lambda}}$ are irreducible for $\bar{\lambda}=0,1,2,8,9,10$, and contain two irreducible subsectors else.

Computing the Hom spaces between the irreducible $\alpha_{\bar{\lambda}}$ shows that they all vanish, except for $\operatorname{Hom}\left(\alpha_{2}, \alpha_{8}\right)$, which is one-dimensional. Hence the two irreducible sectors $\alpha_{2}$ and $\alpha_{8}$ are isomorphic, $\alpha_{2} \cong \alpha_{8}$. Furthermore,

$$
\operatorname{dim} \operatorname{Hom}\left(\alpha_{2}, \alpha_{4}\right)=1=\operatorname{dim} \operatorname{Hom}\left(\alpha_{10}, \alpha_{4}\right),
$$


so that $\alpha_{4} \cong \alpha_{2}+\alpha_{10}$. Similarly, one finds $\alpha_{5} \cong \alpha_{1}+\alpha_{9}$ and $\alpha_{6} \cong \alpha_{0}+\alpha_{2}$. Thus these sectors do not give rise to new irreducible sectors. According to our general conjecture, and in accordance with the results of [6], we expect in total 6 boundary conditions and thus one additional simple object, which we call $\alpha_{3}^{(1)}$. Indeed we have $\operatorname{dim} \operatorname{Hom}_{\mathfrak{A}}\left(\alpha_{3}, \alpha_{\bar{\mu}}\right)=\delta_{\bar{\mu}, 3}+\operatorname{dim} \operatorname{Hom}_{\overline{\mathfrak{A}}}\left(\bar{\Phi}_{\bar{\mu}}, \bar{\Phi}_{6} \star \bar{\Phi}_{3}\right)=\delta_{\bar{\mu}, 3}$ $+\operatorname{dim} \operatorname{Hom}_{\overline{\mathfrak{A}}}\left(\bar{\Phi}_{\bar{\mu}}, \bar{\Phi}_{3}+\bar{\Phi}_{5}+\bar{\Phi}_{7}+\bar{\Phi}_{9}\right)$. So $\alpha_{3}^{(1)}$ appears in the decompositions

$$
\alpha_{3} \cong \alpha_{9}+\alpha_{3}^{(1)} \quad \alpha_{7} \cong \alpha_{1}+\alpha_{3}^{(1)} .
$$

The $\sigma$-restriction is found from formula (7). First,

$$
\sigma\left(\alpha_{0}\right) \cong 0 \oplus 6, \quad \sigma\left(\alpha_{10}\right) \cong 4 \oplus 10, \quad \sigma\left(\alpha_{3}^{(1)}\right) \cong 3 \oplus 7,
$$

showing that these sectors are the three non-solitonic sectors of $B_{2}$ that can already be inferred from the partition function (10), namely

$$
o=\alpha_{0}, \quad v=\alpha_{10}, \quad s=\alpha_{3}^{(1)} .
$$

It is convenient to introduce a similar notation $\check{o}, \check{v}, \check{s}$ for the three solitonic $B_{2}$-sectors; they restrict as follows:

$$
\begin{aligned}
& \sigma(\check{o}) \equiv \sigma\left(\alpha_{1}\right) \cong 1 \oplus 5 \oplus 7, \quad \sigma(\check{v}) \equiv \sigma\left(\alpha_{9}\right) \cong 3 \oplus 5 \oplus 9, \\
& \sigma(\check{s}) \equiv \sigma\left(\alpha_{2}\right) \cong 2 \oplus 4 \oplus 6 \oplus 8 .
\end{aligned}
$$

It is readily checked that all annulus amplitudes reported in [6] can indeed be written as linear combinations of the corresponding six specific sums of $A_{1}$-characters.

The fusion products of the sectors $\alpha_{\bar{\mu}}$ are computed with formula (5). For $o, v$ and $s$ we get the usual Ising fusion rules; they indeed provide the annuli of the Cardy boundary conditions. $\alpha_{0}$ acts generally as the identity under fusion. The remaining fusion rules between ordinary and solitonic sectors turn out to be

$$
\begin{array}{ll}
v \star \check{o}=\check{v}, & v \star \check{v}=\check{o}, \quad v \star \check{s}=\check{s}, \\
s \star \check{o}=\check{s}, \quad s \star \check{v}=\check{s}, \quad s \star \check{s}=\check{o}+\check{v} .
\end{array}
$$

The fusion between two solitonic sectors produces ordinary as well as solitonic sectors; we find

$$
\begin{aligned}
& \check{o} \star \check{o}=o+\check{s}, \quad \check{v} \star \check{v}=o+\check{s}, \\
& \check{o} \star \check{v}=v+\check{s}, \quad \check{v} \star \check{s}=s+\check{o}+\check{v}, \\
& \check{o} \star \check{s}=s+\check{o}+\check{v}, \quad \check{s} \star \check{s}=o+v+2 \check{s} .
\end{aligned}
$$

These fusion products exactly give the annulus multiplicities that have been found by different arguments in [6]. Also the $\tilde{S}$ matrix can be computed. It reads

$$
\tilde{S}=\frac{1}{d}\left(\begin{array}{cccccc}
1 & \sqrt{2} & 1 & \frac{1}{\sqrt{2}}(1+\sqrt{3}) & 1+\sqrt{3} & \frac{1}{\sqrt{2}}(1+\sqrt{3}) \\
\frac{1}{2} d & 0 & -\frac{1}{2} d & \frac{1}{2} d & 0 & -\frac{1}{2} d \\
1 & -\sqrt{2} & 1 & -\frac{1}{\sqrt{2}}(1+\sqrt{3}) & 1+\sqrt{3} & -\frac{1}{\sqrt{2}}(1+\sqrt{3}) \\
\frac{1}{\sqrt{2}}(1+\sqrt{3}) & 1+\sqrt{3} & \frac{1}{\sqrt{2}}(1+\sqrt{3}) & -1 & -\sqrt{2} & -1 \\
\frac{1}{2} d & 0 & -\frac{1}{2} d & -\frac{1}{2} d & 0 & \frac{1}{2} d \\
\frac{1}{\sqrt{2}}(1+\sqrt{3}) & -1-\sqrt{3} & \frac{1}{\sqrt{2}}(1+\sqrt{3}) & 1 & -\sqrt{2} & 1
\end{array}\right)
$$


with $d:=1 / 2 \sqrt{3+\sqrt{3}}$.

Our second example is the exceptional modular invariant of $G_{2}$ at level 3. It reads

$$
Z=\left|\chi_{00}+\chi_{11}\right|^{2}+2\left|\chi_{02}\right|^{2}
$$

and describes the conformal embedding into $E_{6}$ at level 1 . Here $G_{2}$-sectors are characterized by their highest weights. The multiplicity two in the second term of $Z$ indicates that the 27 dimensional irreducible representation of $E_{6}$ and its conjugate restrict to the same irreducible $G_{2}$-representation. $G_{2}$ at level 3 has six irreducible sectors. A careful analysis of the Hom spaces shows that $\alpha_{01}, \alpha_{03}$ and $\alpha_{10}$ are irreducible and all isomorphic. $\alpha_{00}$ is, as always, irreducible, and indeed not isomorphic to $\alpha_{01}$. $\alpha_{11}$ contains two irreducibles, and one finds $\alpha_{11} \cong \alpha_{00}+\alpha_{01}$ so that it does not give rise to any new irreducibles. Finally, $\operatorname{dim} \operatorname{Hom}\left(\alpha_{02}, \alpha_{02}\right)=3$, and $\alpha_{02}$ contains $\alpha_{01}$ as a subobject. We choose the notation $\alpha_{02}^{( \pm)}$for its two other subobjects:

$$
\alpha_{02}=\alpha_{01}+\alpha_{02}^{(+)}+\alpha_{02}^{(-)}
$$

The computation of the $\sigma$-restriction is straightforward, too. We get

$$
\begin{aligned}
& \sigma\left(\alpha_{01}\right)=\bar{\Phi}_{01}+\bar{\Phi}_{02}+\bar{\Phi}_{03}+\bar{\Phi}_{10}+\bar{\Phi}_{11}, \\
& \sigma\left(\alpha_{00}\right)=\bar{\Phi}_{00}+\bar{\Phi}_{11}, \quad \sigma\left(\alpha_{02}\right)=\sigma\left(\alpha_{01}\right)+2 \bar{\Phi}_{02},
\end{aligned}
$$

from which we also learn that $\sigma\left(\alpha_{02}^{( \pm)}\right)=\bar{\Phi}_{02}$. We can therefore identify $\alpha_{00}$ as the vacuum sector of the $E_{6}$-theory and $\alpha_{02}^{( \pm)}$as the sectors corresponding to the two 27-dimensional irreducible representations of $E_{6}$. In addition there is a single solitonic sector, given by $\alpha_{01}$. Notice that we obtain again the same number of simple objects in the bulk and in the boundary category.

It is readily checked that the fusion rules of $\alpha_{00}$ and $\alpha_{02}^{( \pm)}$are indeed the $\mathbb{Z}_{3}$ fusion rules of $E_{6}$ at level 1 . The fusion rules involving $\alpha_{01}$ turn out to be

$$
\begin{aligned}
& \alpha_{01} \star \alpha_{00}=\alpha_{01}, \quad \alpha_{01} \star \alpha_{02}^{(+)}=\alpha_{01}=\alpha_{01} \star \alpha_{02}^{(-)}, \\
& \alpha_{01} \star \alpha_{01}=\alpha_{00}+3 \alpha_{01}+\alpha_{02}^{(+)}+\alpha_{02}^{(-)} .
\end{aligned}
$$

Thus the fusion graph of $\alpha_{01}$ looks like

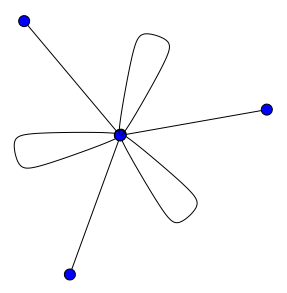

It has already been displayed in [8,9], where also the $\mathbb{Z}_{3}$ fusion rules of the ordinary sectors were established by a different method..

These fusion rules provide the annulus multiplicities. Combining them with the modular $S$-matrix of the $G_{2}$-theory yields three symmetry preserving boundary states,

$$
\begin{aligned}
& \left.\left.\left.\left.|00\rangle=3^{-1 / 4}(|00\rangle\rangle+|11\rangle\right\rangle+|02,+\rangle\right\rangle+|02,-\rangle\right\rangle\right) \\
& \left.\left.\left.\left.|02,+\rangle=3^{-1 / 4}(|00\rangle\rangle+|11\rangle\right\rangle+\mathrm{e}^{2 \pi \mathrm{i} / 3}|02,+\rangle\right\rangle+\mathrm{e}^{-2 \pi \mathrm{i} / 3}|02,-\rangle\right\rangle\right), \\
& \left.\left.\left.\left.|02,-\rangle=3^{-1 / 4}(|00\rangle\rangle+|11\rangle\right\rangle+\mathrm{e}^{-2 \pi \mathrm{i} / 3}|02,+\rangle\right\rangle+\mathrm{e}^{2 \pi \mathrm{i} / 3}|02,-\rangle\right\rangle\right)
\end{aligned}
$$


as well as the single symmetry breaking boundary state

$$
\left.\left.|01\rangle=\frac{1}{2} 3^{1 / 4}(\sqrt{3}+\sqrt{7})|00\rangle\right\rangle+\frac{1}{2} 3^{1 / 4}(\sqrt{3}-\sqrt{7})|11\rangle\right\rangle .
$$

We finally remark that the system of equations for the coefficients of the Ishibashi states is highly over-determined. We regard it as a non-trivial check of our ideas that a solution exists at all.

\section{ACKNOWLEDGEMENT:}

We are grateful to J. Böckenhauer, D.E. Evans, G. Felder, J. Fröhlich and J.-B. Zuber for helpful discussions, and to P. Bantay and B. Schellekens for a careful reading of the manuscript. C.S. would like to thank the Schrödinger Institute for hospitality.

\section{References}

[1] J.L. Cardy, Boundary conditions, fusion rules and the Verlinde formula, Nucl. Phys. B 324 (1989) 581

[2] J. Fuchs and C. Schweigert, Orbifold analysis of broken bulk symmetries, Phys. Lett. B 447 (1999) 266

[3] J. Fuchs and C. Schweigert, Symmetry breaking boundaries I. General theory, Nucl. Phys. B 558 (1999) 419

[4] J. Fuchs and C. Schweigert, Symmetry breaking boundaries II. More structures; examples, Nucl. Phys. B 568 (2000) 543

[5] R.E. Behrend, P.A. Pearce, V.B. Petkova, and J.-B. Zuber, On the classification of bulk and boundary conformal field theories, Phys. Lett. B 444 (1998) 163

[6] R.E. Behrend, P.A. Pearce, V.B. Petkova, and J.-B. Zuber, Boundary conditions in rational conformal field theories, Nucl. Phys. B 570 (2000) 525

[7] V. Pasquier, Two-dimensional critical systems labeled by Dynkin diagrams, Nucl. Phys. B 285 (1987) 162

[8] P. di Francesco and J.-B. Zuber, $S U(N)$ Lattice integrable models and modular invariance, in: Recent Developments in CFT, E. Gava et al., eds. (World Scientific, Singapore 1989)

[9] P. di Francesco, Integrable lattice models, graphs and modular invariant conformal field theories, Int. J. Mod. Phys. A 7 (1992) 407

[10] V.B. Petkova and J.-B. Zuber, From CFT's to graphs, Nucl.Phys. B 463 (1996) 161

[11] R. Longo and K.-H. Rehren, Nets of subfactors, Rev. Math. Phys. 7 (1995) 567

[12] F. Xu, New braided endomorphisms from conformal inclusions, Commun. Math. Phys. 192 (1998) 349

[13] J. Böckenhauer and D.E. Evans, Modular invariants, graphs, and $\alpha$-induction for nets of subfactors I, II, III, Commun. Math. Phys. 197 (1998) 361, 200 (1999) 57, 205 (1999) 183

[14] J. Böckenhauer, D.E. Evans, and Y. Kawahigashi, Chiral structure of modular invariants for subfactors, Commun. Math. Phys. 210 (2000) 733

[15] J. Böckenhauer, D.E. Evans, and Y. Kawahigashi, Longo-Rehren subfactors arising from $\alpha$-induction, preprint math.OA/0002154 
[16] A.N. Schellekens and S. Yankielowicz, Simple currents, modular invariants, and fixed points, Int. J. Mod. Phys. A 5 (1990) 2903

[17] J. Lepowsky, Calculus of twisted vertex operators, Proc. Natl. Acad. Sci. USA 82 (1985) 8295

[18] I.B. Frenkel, J. Lepowsky, and A. Meurman, Vertex Operator Algebras and the Monster (Academic Press, New York 1988)

[19] C. Dong, Twisted modules for vertex algebras associated with even lattices, J. Algebra 165 (1994) 91

[20] C. Dong, H. Li, and G. Mason, Twisted representations of vertex operator algebras, Math. Annal. 310 (1998) 571

[21] M.R. Gaberdiel, Fusion of twisted representations, Int. J. Mod. Phys. A 12 (1997) 5183

[22] G. Felder, J. Fröhlich, J. Fuchs, and C. Schweigert, Conformal boundary conditions and threedimensional topological field theory, Phys. Rev. Lett. 84 (2000) 1659

[23] G. Felder, J. Fröhlich, J. Fuchs, and C. Schweigert, Correlation functions and boundary conditions in RCFT and three-dimensional topology, preprint hep-th/9912239

[24] J. Fröhlich, New superselection sectors ('soliton sectors') in two-dimensional Bose quantum field models, Commun. Math. Phys. 47 (1976) 269

[25] K. Fredenhagen, Superselection sectors in low dimensional quantum field theory, J. Geom. and Phys. 11 (1993) 337

[26] M. Müger, On soliton automorphisms in massive and conformal theories, Rev. Math. Phys. 11 (1999) 337

[27] V.G. Turaev, Quantum Invariants of Knots and 3-Manifolds (de Gruyter, New York 1994)

[28] K. Fredenhagen, K.-H. Rehren, and B. Schroer, Superselection sectors with braid group statistics and exchange algebras, I: General theory, Commun. Math. Phys. 125 (1989) 201

[29] J. Fröhlich and F. Gabbiani, Braid statistics in local quantum theory, Rev. Math. Phys. 2 (1990) 251

[30] R. Haag, Local Quantum Physics (Springer Verlag, Berlin 1992)

[31] F. Gabbiani and J. Fröhlich, Operator algebras and conformal field theory, Commun. Math. Phys. 155 (1993) 569

[32] R. Brunetti, D. Guido, and R. Longo, Modular structure and duality in conformal quantum field theory, Commun. Math. Phys. 156 (1993) 201

[33] F. Xu, Algebraic coset conformal field theories, Commun. Math. Phys. 211 (2000) 1

[34] F. Xu, Algebraic orbifold conformal field theories, preprint math.QA/0004150

[35] A. Bruguières, Catégories prémodulaires, modularisations et invariants des variétés de dimension 3, Math. Annal. 316 (2000) 215

[36] M. Müger, Galois theory for braided tensor categories and the modular closure, Adv. Math. 150 (2000) 151

[37] S. Sawin, Jones-Witten invariants for nonsimply-connected Lie groups and the geometry of the Weyl alcove, preprint math.QA/9905010

[38] J. Fuchs, A.N. Schellekens, and C. Schweigert, A matrix $S$ for all simple current extensions, Nucl. Phys. B 473 (1996) 323

[39] R. Longo and J.E. Roberts, A theory of dimension, K-Theory 11 (1997) 103 
[40] L.R. Huiszoon and A.N. Schellekens, Crosscaps, boundaries and T-Duality, preprint hepth/0004100

[41] G. Pradisi, A. Sagnotti, and Ya.S. Stanev, Planar duality in SU(2) WZW models, Phys. Lett. B 354 (1995) 279 